\title{
A characterization of seminormal C-monoids
}

\author{
Alfred Geroldinger $^{1}(\mathbb{1}) \cdot$ Qinghai Zhong $^{1}$
}

Received: 27 September 2018 / Accepted: 30 January 2019 / Published online: 9 February 2019

(c) The Author(s) 2019

\begin{abstract}
It is well-known that a C-monoid is completely integrally closed if and only if its reduced class semigroup is a group and if this holds, then the C-monoid is a Krull monoid and the reduced class semigroup coincides with the usual class group of Krull monoids. We prove that a $\mathrm{C}$-monoid is seminormal if and only if its reduced class semigroup is a union of groups. Based on this characterization we establish a criterion (in terms of the class semigroup) when seminormal C-monoids are half-factorial.
\end{abstract}

Keywords Krull monoids · C-monoids $\cdot$ Seminormal $\cdot$ Class semigroups $\cdot$ Half-factorial

Mathematics Subject Classification $20 \mathrm{M} 13 \cdot 13 \mathrm{~A} 05 \cdot 13 \mathrm{~A} 15 \cdot 13 \mathrm{~F} 05 \cdot 13 \mathrm{~F} 45$

\section{Introduction}

A C-monoid $H$ is a submonoid of a factorial monoid, say $H \subset F$, such that $H^{\times}=H \cap F^{\times}$ and the reduced class semigroup is finite. A commutative ring is a $\mathrm{C}$-ring if its multiplicative monoid of regular elements is a C-monoid. Every C-monoid is Mori (i.e., $v$-noetherian), its complete integral closure $\widehat{H}$ is a Krull monoid with finite class group $\mathcal{C}(\widehat{H})$, and the conductor $(H: \widehat{H})$ is non-trivial. Conversely, every Mori domain $R$ with non-zero conductor $\mathfrak{f}=(R: \widehat{R})$, for which the residue class ring $\widehat{R} / \mathfrak{f}$ and the class group $\mathcal{C}(\widehat{R})$ are finite, is a C-domain ([10, Theorem 2.11.9]), and these two finiteness conditions are equivalent to being a C-domain for non-local semilocal noetherian domains ([23, Corollary 4.5]). The following result is well-known (see Sect. 2 where we gather the basics on C-monoids).

This work was supported by the Austrian Science Fund FWF, Project number P28864-N35.

$\triangle \quad$ Alfred Geroldinger

alfred.geroldinger@uni-graz.at

https://imsc.uni-graz.at/geroldinger

Qinghai Zhong

qinghai.zhong@uni-graz.at

https://imsc.uni-graz.at/zhong/

1 Institute for Mathematics and Scientific Computing, NAWI Graz, University of Graz, Heinrichstraße 36, 8010 Graz, Austria 
Theorem A Let $H$ be a C-monoid. Then $H$ is completely integrally closed if and only if its reduced class semigroup is a group. If this holds, then $H$ is a Krull monoid and the reduced class semigroup coincides with the class group of $H$.

The goal of the present note is the following characterization of seminormal C-monoids. Note that all seminormal C-domains are seminormal Mori domains (see [2] for a survey) and that, in particular, all seminormal orders in algebraic number fields (see [7]) are seminormal C-domains. Moreover, the seminormalization of any C-monoid is a seminormal C-monoid (Proposition 3.1).

Theorem 1.1 Let $H$ be a $C$-monoid. Then $H$ is seminormal if and only if its reduced class semigroup is a union of groups. If this holds, then we have

1. The class of every element of $H$ is an idempotent of the class semigroup.

2. The constituent group of the smallest idempotent of the class semigroup (with respect to the Rees order) is isomorphic to the class group of the complete integral closure of $H$.

The finiteness of the class semigroup allows to establish a variety of arithmetical finiteness results for C-monoids (for a sample, out of many, see [10, Theorem 4.6.6] or [8]). In the special case of Krull monoids with finite class group, precise arithmetical results can be given in terms of the structure of the class group (for a survey see [25]). As a first step in this direction in the more general case of seminormal C-monoids, we establish—based on Theorem 1.1-a characterization of half-factoriality in terms of the class semigroup (Theorem 4.2).

\section{Background on C-monoids}

We denote by $\mathbb{N}$ the set of positive integers and set $\mathbb{N}_{0}=\mathbb{N} \cup\{0\}$. For rationals $a, b \in \mathbb{Q}$, we denote by $[a, b]=\{x \in \mathbb{Z} \mid a \leq x \leq b\}$ the discrete interval lying between $a$ and $b$. All semigroups and rings in this paper are commutative and have an identity element and all homomorphisms respect identity elements. Let $S$ be a multiplicatively written semigroup. Then $S^{\times}$denotes its group of invertible elements and $\mathrm{E}(S)$ its set of idempotents. For subsets $A, B \subset S$ and an element $c \in S$ we set

$$
A B=\{a b \mid a \in A, b \in B\} \text { and } c B=\{c b \mid b \in B\} .
$$

Let $\mathcal{C}$ be a semigroup. We use additive notation and have class groups and class semigroups in mind. Let $e, f \in \mathrm{E}(\mathcal{C})$. We denote by $\mathcal{C}_{e}$ the set of all $x \in \mathcal{C}$ such that $x+e=x$ and $x+y=e$ for some $y \in \mathcal{C}$. Then $\mathcal{C}_{e}$ is a group with identity element $e$, called the constituent group of $e$. If $e \neq f$, then $\mathcal{C}_{e} \cap \mathcal{C}_{f}=\emptyset$. An element $x \in \mathcal{C}$ is contained in a subgroup of $\mathcal{C}$ if and only if there is some $e \in \mathrm{E}(\mathcal{C})$ such that $x \in \mathcal{C}_{e}$. Thus $\mathcal{C}$ is a union of groups if and only if

$$
\mathcal{C}=\bigcup_{e \in \mathrm{E}(\mathcal{C})} \mathcal{C}_{e}
$$

Semigroups with this property are called Clifford semigroups $([15,16])$. Clearly, $0 \in \mathrm{E}(\mathcal{C})$ and $\mathcal{C}_{0}=\mathcal{C}^{\times}$. The Rees order $\leq$on $\mathrm{E}(S)$ is defined by $e \leq f$ if $e+f=e$. If $\mathrm{E}(S)=\{0=$ $\left.e_{0}, \ldots, e_{n}\right\}$, then $e_{0}$ is the largest element and $e_{0}+\cdots+e_{n}$ is the smallest idempotent in the Rees order. If $\mathcal{C}$ is finite, then for every $a \in \mathcal{C}$ there is an $n \in \mathbb{N}$ such that $n a=a+\cdots+a \in$ $\mathrm{E}(\mathcal{C})$. 
By a monoid, we mean a cancellative semigroup. We use multiplicative notation and have monoids of nonzero elements of domains in mind. Let $H$ be a monoid, $H_{\text {red }}=\left\{a H^{\times} \mid a \in\right.$ $H\}$ the associated reduced monoid, and $\mathrm{q}(H)$ the quotient group of $H$. We denote by

- $H^{\prime}=\left\{x \in \mathrm{q}(H) \mid\right.$ there is an $m \in \mathbb{N}$ such that $x^{n} \in H$ for all $\left.n \geq m\right\}$ the seminormalization,

- $\widetilde{H}=\left\{x \in \mathrm{q}(H) \mid\right.$ there is an $N \in \mathbb{N}$ such that $\left.x^{N} \in H\right\}$ the root closure, and by

- $\widehat{H}=\left\{x \in \mathrm{q}(H) \mid\right.$ there is a $c \in H$ such that $c x^{n} \in H$ for all $\left.n \in \mathbb{N}\right\}$ the complete integral closure of $H$.

Then $H \subset H^{\prime} \subset \widetilde{H} \subset \widehat{H} \subset \mathrm{q}(H)$ and $H$ is said to be

- seminormal if $H=H^{\prime}$,

- root closed if $H=\widetilde{H}$,

- completely integrally closed if $H=\widehat{H}$,

- $v$-noetherian (or Mori) if $\mathrm{H}$ satisfies the ACC on divisorial ideals,

- a Krull monoid if $\mathrm{H}$ is a completely integrally closed Mori monoid.

Let $F$ be a factorial monoid, say $F=F^{\times} \times \mathcal{F}(P)$, where $\mathcal{F}(P)$ is the free abelian monoid with basis $P$. Then every $a \in F$ can be written in the form $a=\varepsilon \prod_{p \in P} p^{\mathrm{v}_{p}(a)}$, where $\varepsilon \in F^{\times}$and $\mathrm{v}_{p}: F \rightarrow \mathbb{N}_{0}$ is the $p$-adic exponent. Let $H \subset F$ be a submonoid. Two elements $y, y^{\prime} \in F$ are called $H$-equivalent (we write $y \sim y^{\prime}$ ) if $y^{-1} H \cap F=y^{\prime-1} H \cap F$ or, in other words,

$$
\text { if for all } x \in F \text {, we have } x y \in H \text { if and only if } x y^{\prime} \in H \text {. }
$$

$H$-equivalence defines a congruence relation on $F$ and for $y \in F$, let $[y]_{H}^{F}=[y]$ denote the congruence class of $y$. Then

$$
\mathcal{C}(H, F)=\{[y] \mid y \in F\} \quad \text { and } \mathcal{C}^{*}(H, F)=\left\{[y] \mid y \in\left(F \backslash F^{\times}\right) \cup\{1\}\right\}
$$

are commutative semigroups with identity element [1] (introduced in [9, Section 4]). $\mathcal{C}(H, F)$ is the class semigroup of $H$ in $F$ and the subsemigroup $\mathcal{C}^{*}(H, F) \subset \mathcal{C}(H, F)$ is the reduced class semigroup of $H$ in $F$. We have

$$
\mathcal{C}(H, F)=\mathcal{C}^{*}(H, F) \cup\left\{[y] \mid y \in F^{\times}\right\} \quad \text { and } \quad\left\{[y] \mid y \in F^{\times}\right\} \cong F^{\times} / H^{\times} .
$$

It is easy to check that either $\left\{[y] \mid y \in F^{\times}\right\} \subset \mathcal{C}^{*}(H, F)$ or $\mathcal{C}^{*}(H, F) \cap\left\{[y] \mid y \in F^{\times}\right\}=$ $\{[1]\}$. Thus

$$
\mathcal{C}(H, F) \text { is a union of groups if and only if } \mathcal{C}^{*}(H, F) \text { is a union of groups }
$$

and $\mathcal{C}(H, F)$ is finite if and only if both $\mathcal{C}^{*}(H, F)$ and $F^{\times} / H^{\times}$are finite. As usual, (reduced) class semigroups and class groups will be written additively.

A monoid homomorphism $\partial: H \rightarrow \mathcal{F}(P)$ is called a divisor theory (for $H$ ) if the following two conditions hold:

- If $a, b \in H$ and $\varphi(a) \mid \varphi(b)$ in $\mathcal{F}(P)$, then $a \mid b$ in $H$.

- For every $\alpha \in \mathcal{F}(P)$ there are $a_{1}, \ldots, a_{m} \in H$ such that $\alpha=\operatorname{gcd}\left(\varphi\left(a_{1}\right), \ldots, \varphi\left(a_{m}\right)\right)$.

A monoid has a divisor theory if and only if it is a Krull monoid ( [10, Theorem 2.4.8]). Suppose $H$ is a Krull monoid. Then there is an embedding of $H_{\text {red }}$ into a free abelian monoid, say $H_{\text {red }} \hookrightarrow \mathcal{F}(P)$. In this case $\mathcal{F}(P)$ is uniquely determined (up to isomorphism) and

$$
\mathcal{C}(H)=\mathrm{q}(\mathcal{F}(P)) / \mathrm{q}\left(H_{\text {red }}\right)
$$


is the (divisor) class group of $H$ which is isomorphic to the $v$-class group $\mathcal{C}_{v}(H)$ of $H$ ([10, Sections 2.1 and 2.4]).

A monoid $H$ is a C-monoid if it is a submonoid of some factorial monoid $F=F^{\times} \times \mathcal{F}(P)$ such that $H^{\times}=H \cap F^{\times}$and the reduced class semigroup is finite. We say that $H$ is dense in $F$ if $\mathrm{v}_{p}(H) \subset \mathbb{N}_{0}$ is a numerical monoid for all primes $p$ of $F$. Suppose that $H$ is a $\mathrm{C}$-monoid. Proofs of the following facts can be found in ([10, Theorems 2.9.11 and 2.9.12]). The monoid $H$ is $v$-noetherian, the conductor $(H: \widehat{H}) \neq \emptyset$, and there is a factorial monoid $F$ such that $H \subset F$ is dense. Suppose that $H \subset F$ is dense. Then the map

$$
\partial: \widehat{H} \rightarrow \mathcal{F}(P), \quad \text { defined by } \quad \partial(a)=\prod_{p \in P} p^{\mathrm{v}_{p}(a)}
$$

is a divisor theory. In particular, $\widehat{H}$ is a Krull monoid, $F_{\text {red }}$ and hence $\mathcal{C}^{*}(H, F)$ are uniquely determined by $H$, and we call $\mathcal{C}^{*}(H, F)$ the reduced class semigroup of $H$.

$$
\text { If } \mathcal{C}^{*}(H, F) \text { is a group, then } H \text { is a Krull monoid }
$$

and every Krull monoid with finite class group is a C-monoid.

Let $R$ be a (commutative integral) domain. Then $R$ is seminormal (completely integrally closed, Mori) if and only if its multiplicative monoid $R^{\bullet}$ of nonzero elements has the respective property. Moreover, $R$

- is a Krull domain if $R^{\bullet}$ is a Krull monoid, and

- is a $C$-domain if $R^{\bullet}$ is a C-monoid.

Both statements generalize to rings with zero-divisors ( [12, Theorem 3.5 and Section 4]). We refer to $[1,6,17,20]$ for C-monoids, that do not stem from ring theory, and to [18] for a more general concept.

\section{A characterization of seminormality}

In this section we first show that the seminormalization and the root closure of $\mathrm{C}$-monoids are still C-monoids. Since the seminormalization of a monoid is seminormal and the root closure is root closed and hence seminormal, Proposition 3.1 shows that the natural closure operations, seminormalization and root closure, of C-monoids lead to seminormal C-monoids. Then we prove the characterization of seminormality, as formulated in Theorem 1.1. After that we discuss a special class of C-monoids, namely the monoid of product-one sequences over a finite group.

Proposition 3.1 Let $H \subset F=F^{\times} \times \mathcal{F}(P)$ be a $C$-monoid. Then the canonical maps

$$
\psi^{\prime}: \mathcal{C}^{*}(H, F) \rightarrow \mathcal{C}^{*}\left(H^{\prime}, F\right) \text { and } \widetilde{\psi}: \mathcal{C}^{*}(H, F) \rightarrow \mathcal{C}^{*}(\tilde{H}, F),
$$

mapping $[a]_{H}^{F} \mapsto[a]_{H^{\prime}}^{F}$ and $[a]_{H}^{F} \mapsto[a]_{\widetilde{H}}^{F}$ for every $a \in F$, are epimorphism with $\psi^{\prime}\left(\mathcal{C}^{*}(H, F)\right)=\mathcal{C}^{*}\left(H^{\prime}, F\right)$ and $\widetilde{\psi}\left(\mathcal{C}^{*}(H, F)\right)=\mathcal{C}^{*}(\widetilde{H}, F)$. Moreover, $H^{\prime} \subset F$ is a seminormal $C$-monoid and $\widetilde{H} \subset F$ is a root closed $C$-monoid. If every class of $\mathcal{C}^{*}(H, F)$ contains an element from $P$, then every class of $\mathcal{C}^{*}\left(H^{\prime}, F\right)$ and every class of $\mathcal{C}^{*}(\widetilde{H}, F)$ contains an element from $P$.

Proof We have $H \subset H^{\prime} \subset \widetilde{H} \subset F, H^{\prime}$ is seminormal, $\widetilde{H}$ is root closed, $H^{\times}=H \cap F^{\times}$, $\left(H^{\prime}\right)^{\times} \subset H^{\prime} \cap F^{\times}$, and $(\widetilde{H})^{\times} \subset \widetilde{H} \cap F^{\times}$. Let $a \in H^{\prime} \cap F^{\times}$. Then $a^{k} \in H \cap F^{\times}=H^{\times}$ 
for some $k \in \mathbb{N}$ and hence there exists $b \in H^{\times} \subset H^{\prime}$ such that $a^{k} b=1$. Since $a^{k-1} b \in H^{\prime}$, we obtain that $a \in\left(H^{\prime}\right)^{\times}$. Therefore $\left(H^{\prime}\right)^{\times}=H^{\prime} \cap F^{\times}$. Let $a \in \widetilde{H} \cap F^{\times}$. Then $a^{k} \in$ $H \cap F^{\times}=H^{\times}$for some $k \in \mathbb{N}$ and hence there exists $b \in H^{\times} \subset \widetilde{H}$ such that $a^{k} b=1$. Since $a^{k-1} b \in \widetilde{H}$, we obtain that $a \in(\widetilde{H})^{\times}$. Therefore $(\widetilde{H})^{\times}=\widetilde{H} \cap F^{\times}$.

To verify that $\psi^{\prime}$ is well-defined, let $a, b \in F$ with $[a]_{H}^{F}=[b]_{H}^{F}$. For an $x \in F$ with $a x \in H^{\prime}$ we have to verify that $b x \in H^{\prime}$. Then this implies that $[a]_{H^{\prime}}^{F}=[b]_{H^{\prime}}^{F}$. In fact, since $a x \in H^{\prime}$, there exists $N \in \mathbb{N}$ such that for all $n \geq N$, we have $(a x)^{n} \in H$. Since $[a]_{H}^{F}=[b]_{H}^{F}$, it follows that $\left[a^{n}\right]_{H}^{F}=\left[b^{n}\right]_{H}^{F}$ whence $a^{n} x^{n} \in H$ implies that $b^{n} x^{n} \in H$. Therefore for all $n \geq N$, we have $(b x)^{n} \in H$ which implies that $b x \in H^{\prime}$.

To verify that $\widetilde{\psi}$ is well-defined, let $a, b \in F$ with $[a]_{H}^{F}=[b]_{H}^{F}$ and let $x \in F$ such that $a x \in \widetilde{H}$. Since $a x \in \widetilde{H}$, there exists $N \in \mathbb{N}$ such that $(a x)^{N} \in H$. Since $[a]_{H}^{F}=[b]_{H}^{F}$, it follows that $\left[a^{N}\right]_{H}^{F}=\left[b^{N}\right]_{H}^{F}$ whence $a^{N} x^{N} \in H$ implies that $b^{N} x^{N} \in H$. Therefore $b x \in \widetilde{H}$.

Obviously, $\psi^{\prime}$ and $\widetilde{\psi}$ are epimorphisms with

$$
\begin{aligned}
\psi^{\prime}\left(\mathcal{C}^{*}(H, F)\right) & =\psi^{\prime}\left(\left\{[a]_{H}^{F} \mid a \in F \backslash F^{\times} \cup\{1\}\right\}\right)=\left\{[a]_{H^{\prime}}^{F} \mid a \in F \backslash F^{\times} \cup\{1\}\right\}=\mathcal{C}^{*}\left(H^{\prime}, F\right) \text { and } \\
\widetilde{\psi}\left(\mathcal{C}^{*}(H, F)\right) & =\widetilde{\psi}\left(\left\{[a]_{H}^{F} \mid a \in F \backslash F^{\times} \cup\{1\}\right\}\right)=\left\{[a]_{\widetilde{H}}^{F} \mid a \in F \backslash F^{\times} \cup\{1\}\right\}=\mathcal{C}^{*}(\widetilde{H}, F) .
\end{aligned}
$$

Thus $H^{\prime} \subset F$ is a seminormal C-monoid and $\widetilde{H} \subset F$ is a root closed C-monoid.

Proof of Theorem 1.1 By [10, Theorem 2.9.11.4], we may choose a special factorial monoid $F$ such that $H \subset F$ is a dense $\mathrm{C}$-monoid. This special choice will turn out to be useful in the proof of part 3. Since $\widehat{H}$ is a Krull monoid, we have $\widehat{H}=\widehat{H}^{\times} \times H_{0}$, where $H_{0}$ is a reduced Krull monoid. If the embedding $H_{0} \hookrightarrow \mathcal{F}(P)$ is a divisor theory, then $H \subset F=\widehat{H}^{\times} \times \mathcal{F}(P)$ is a dense C-monoid. In particular, we have $H^{\times}=H \cap F^{\times}$and $\mathcal{C}^{*}=\mathcal{C}^{*}(H, F)$ is finite. Furthermore, there are $\alpha \in \mathbb{N}$ and a subgroup $V \subset F^{\times}$such that the following properties hold ([10, Proposition 2.8.11 and Definition 2.9.5]):

P1. $H^{\times} \subset V, \quad\left(F^{\times}: V\right) \mid \alpha, \quad V\left(H \backslash H^{\times}\right) \subset H$, P2. $q^{2 \alpha} F \cap H=q^{\alpha}\left(q^{\alpha} F \cap H\right)$ for all $q \in F \backslash F^{\times}$.

In particular, if $p \in P$ and $a \in p^{\alpha} F$, then $a \in H$ if and only if $p^{\alpha} a \in H$.

1. First we suppose that $H$ is seminormal. We have to show that $\mathcal{C}^{*}$ is a union of groups. We choose an element $a \in F \backslash F^{\times}$, say $a=\epsilon p_{1}^{k_{1}} \cdot \ldots \cdot p_{t}^{k_{t}}$, where $\epsilon \in F^{\times}, p_{1}, \ldots, p_{t} \in P$, $t, k_{1}, \ldots, k_{t} \in \mathbb{N}$. Since $\mathcal{C}^{*}$ is finite, there is an $n \in \mathbb{N}$ such that $\left[a^{n}\right]$ is an idempotent element of $\mathcal{C}^{*}$. Since for every multiple $n^{\prime}$ of $n,\left[a^{n^{\prime}}\right]$ is an idempotent element of $\mathcal{C}^{*}$, we may assume without restriction that $n$ is a multiple of $\alpha$. We assert that $a^{n+1} \sim a$. Clearly, this implies that $[a]$ lies in a cyclic subgroup of $\mathcal{C}^{*}$.

In order to show that $a^{n+1} \sim a$, we choose an element $b \in F$. First, suppose that $a b \in H$. Then for every $r \geq \alpha$, we have $(a b)^{r} \in H \cap p_{1}^{\alpha} \cdot \ldots \cdot p_{t}^{\alpha} F$. It follows by Properties (P1) and (P2) that $\epsilon^{n}=\left(\epsilon^{\alpha}\right)^{\frac{n}{\alpha}} \in V$ and $p_{j}^{\alpha}(a b)^{r} \in H \backslash H^{\times}$for all $j \in[1, t]$. Using Property (P2) repeatedly we infer that

$$
\left(a^{n+1} b\right)^{r}=a^{r n}(a b)^{r}=\epsilon^{r n} p_{1}^{r k_{1}(n / \alpha) \alpha} \cdot \ldots \cdot p_{t}^{r k_{t}(n / \alpha) \alpha}(a b)^{r} \in V\left(H \backslash H^{\times}\right) \subset H .
$$

Since $H$ is seminormal, we obtain $a^{n+1} b \in H$.

Conversely, suppose that $b a^{n+1} \in H$. We have to verify that $b a \in H$. To do so we claim that

$$
(b a)^{m} a^{n} \in H \text { for all } m \in \mathbb{N},
$$


and we proceed by induction on $m$. If $m=1$, then this holds by assumption. Suppose the claim holds for $m \in \mathbb{N}$. Then, by the induction hypothesis,

$$
(b a)^{m+1} a^{n} \sim(b a)^{m+1} a^{2 n}=\left((b a)^{m} a^{n}\right)\left(b a^{n+1}\right) \in H
$$

whence $(b a)^{m+1} a^{n} \in H$. Using (3.1) with $m=n$ we infer that

$$
(b a)^{n}=b^{n} a^{n} \sim b^{n} a^{2 n}=(b a)^{n} a^{n} \in H
$$

whence $(b a)^{n} \in H$. Thus we obtain that

$$
(b a)^{n+1}=\left(b^{n+1} a\right) a^{n} \sim\left(b^{n+1} a\right) a^{2 n}=(b a)^{n}\left(b a^{n+1}\right) \in H
$$

whence $(b a)^{n+1} \in H$. This implies that $(b a)^{\ell} \in H$ for all sufficiently large $\ell \in \mathbb{N}$. Since $H$ is seminormal, it follows that $b a \in H$.

2. Now we suppose that $\mathcal{C}^{*}$ is a union of groups. Then, by (2.2), the class semigroup $\mathcal{C}(H, F)$ is a union of groups. In order to show that $H$ is seminormal, let $a \in \mathrm{q}(H)$ be given such that $a^{n} \in H$ for all $n \geq N$ for some $N \in \mathbb{N}$. Since $F$ is factorial, it is seminormal whence $H \subset F$ and $\mathrm{q}(H) \subset \mathrm{q}(F)$ imply that $a \in F$. If $a \in F^{\times}$, then $a=a^{N+1}\left(a^{N}\right)^{-1} \in H$. Suppose $a \in F \backslash F^{\times}$. Since $[a] \in \mathcal{C}^{*}$ and $\mathcal{C}^{*}$ is finite, we obtain that $\langle[a]\rangle \subset \mathcal{C}^{*}$ is a finite cyclic group. If $N_{0} \in \mathbb{N}$ is the order of $[a]$ in the cyclic group $\langle[a]\rangle \subset \mathcal{C}^{*}$, then $a \sim a^{N_{0} t+1}$ for all $t \in \mathbb{N}$. Since $N_{0} N+1>N$, we obtain that $a^{N_{0} N+1} \in H$ and hence $a \in H$.

3. Suppose that $H$ is seminormal and set $\mathrm{E}\left(\mathcal{C}^{*}\right)=\left\{e_{0}, \ldots, e_{n}\right\}$, where $n \in \mathbb{N}_{0}, e_{0}=[1]$, and $e_{0}+\cdots+e_{n}=e_{n}$. For $i \in[0, n]$, we set $\mathcal{C}_{i}^{*}=\mathcal{C}_{e_{i}}^{*}$. Since $\mathcal{C}^{*}$ is a union of groups, (2.1) implies that

$$
\mathcal{C}^{*}=\biguplus_{i=0}^{n} \mathcal{C}_{i}^{*} .
$$

For every $i \in[0, n]$, we have $\mathcal{C}_{i}^{*}=\left\{g \in \mathcal{C}^{*} \mid m g=e_{i}\right.$ for some $\left.m \in \mathbb{N}\right\}$ and $\mathcal{C}_{i}^{*}+C_{n}^{*}=C_{n}^{*}$.

For the class group of the Krull monoid $\widehat{H}$, we have

$$
\mathcal{C}(\widehat{H})=\mathrm{q}(\mathcal{F}(P)) / \mathrm{q}\left(H_{0}\right)=\mathrm{q}(F) / \mathrm{q}(\widehat{H})=\mathrm{q}(F) / \mathrm{q}(H) .
$$

Since $H_{0} \hookrightarrow \mathcal{F}(P)$ is a divisor theory, the inclusion $\widehat{H}=\widehat{H}^{\times} \times H_{0} \subset F=\widehat{H}^{\times} \times \mathcal{F}(P)$ is saturated whence $\widehat{H}=\mathrm{q}(\widehat{H}) \cap F=\mathrm{q}(H) \cap F$ ([10, Corollary 2.4.3.2]).

3.(a) Let $a \in H$. If $a \in H^{\times}$, then [a] $=[1]$ is an idempotent of $\mathcal{C}^{*}$. Suppose $a \in H \backslash H^{\times}$. We have to show that $a \sim a^{2}$ and to do so we choose some $b \in F$. If $a b \in H$, then obviously $a^{2} b \in H$. Conversely, suppose that $a^{2} b \in H$. Since $\mathcal{C}^{*}$ is a finite Clifford semigroup, there exists an $m \in \mathbb{N}$ such that $a^{m+1} \sim a$. Then $a b \sim a^{m+1} b=a^{m-1} a^{2} b \in H$ which implies $a b \in H$.

3.(b) By [10, Proposition 2.8.7.1], the map $\Phi: \mathcal{C}^{*} \rightarrow \mathcal{C}(\widehat{H})$ given by $\Phi([a])=a \mathrm{q}(\widehat{H})$ is an epimorphism. Therefore $\Phi_{n}=\left.\Phi\right|_{\mathcal{C}_{n}^{*}}: \mathcal{C}_{n}^{*} \rightarrow \mathcal{C}(\widehat{H})$ is a group homomorphism, and it remains to show that it is bijective.

Let $b \in F \backslash F^{\times}$such that $[b]=e_{n}$. Since $e_{n}$ is the identity of $\mathcal{C}_{n}^{*}, \Phi_{n}\left(e_{n}\right)$ is the identity element of $\mathcal{C}(\widehat{H})$ whence $b \mathrm{q}(\widehat{H})=\Phi_{n}\left(e_{n}\right)=\mathrm{q}(H)$ and $b \in \mathrm{q}(H) \cap F=\widehat{H}$. Then for every $a \in F$ we have $[b a]=[b]+[a] \in \mathcal{C}_{n}^{*}$ and $\Phi([b a])=b a \mathrm{q}(\widehat{H})=a \mathrm{q}(\widehat{H})$. Thus $\Phi_{n}$ is surjective.

Let $a \in F \backslash F^{\times}$with $[a] \in \mathcal{C}_{n}^{*}$ such that $\Phi_{n}([a])$ is the identity element of $\mathcal{C}(\widehat{H})$. Then $a \mathrm{q}(H)=\mathrm{q}(H)$ and $a \in \mathrm{q}(H) \cap F=\widehat{H}$ whence there exists $c \in H$ such that $c a \in H$. Thus 3.(a) implies that $[\mathrm{ca}]$ and $[c]$ are idempotents. Since $[a]$ and $e_{n}$ are in $\mathcal{C}_{n}^{*}$ and $C_{i}^{*}+C_{n}^{*}=C_{n}^{*}$ 
for all $i \in[0, n]$, it follows that $[c a]=[c]+[a]$ and $[c]+e_{n}$ are in $\mathcal{C}_{n}^{*}$. Since $[c a]=[c a]+[c a]$, it follows that $[c a]=e_{n}$ and similarly $[c]+e_{n}=e_{n}$. Thus we obtain that

$$
[a]=[a]+e_{n}=[a]+[c]+e_{n}=[c a]+e_{n}=e_{n},
$$

which implies that $\Phi_{n}$ is injective.

In our next remark and also in Sect. 4, we need the concept of monoids of product-one sequences over groups. Let $G$ be a finite group and $\mathcal{F}(G)$ be the free abelian monoid with basis $G$. An element $S=g_{1} \cdot \ldots \cdot g_{\ell} \in \mathcal{F}(G)$ is said to be a product-one sequence (over $G$ ) if its terms can be ordered such that their product equals $1_{G}$, the identity element of the group $G$. The monoid $\mathcal{B}(G)$ of all product-one sequences over $G$ is a finitely generated C-monoid, and it is a Krull monoid if and only if $G$ is abelian ([6, Theorem 3.2]).

\section{Remark 3.2}

1. The main statement of Theorem 1.1 was proved first for the monoid of product-one sequences over a finite group. Let $G$ be a finite group and $G^{\prime}$ be its commutator subgroup. Jun Seok Oh showed that $\mathcal{B}(G)$ is seminormal if and only if $\left|G^{\prime}\right| \leq 2$ if and only if its class semigroup $\mathcal{C}^{*}(\mathcal{B}(G), \mathcal{F}(G))$ is a union of groups ([21, Corollary 3.12]).

2. Let $H \subset F$ be a $\mathrm{C}$-monoid with all conventions as in Theorem 1.1. If $a \in H$, then Theorem 1.1.1 implies that the element $[a] \in \mathcal{C}(H, F)$ is idempotent. In case $H=$ $\mathcal{B}(G)$, the converse holds and this fact was used in the characterization when $\mathcal{B}(G)$ is seminormal. However, the converse does not hold true for general $\mathrm{C}$-monoids as the next example shows.

3. Let $F=\mathcal{F}(P)$ with $P=\left\{p_{1}, p_{2}\right\}$ and

$$
H=\left[p_{1} p_{2}, p_{1}^{2 k} p_{2} \mid k \geq 0\right]=\left\{1, p_{1} p_{2}\right\} \cup\left\{p_{1}^{2 k} p_{2} \mid k \geq 0\right\} \cup\left\{p_{1}^{t} p_{2}^{s} \mid t \geq 0, s \geq 2\right\} \subset F .
$$

Then $\left(H: p_{1}^{2}\right)=\left(H: p_{1}^{4}\right)=H \backslash\left\{1, p_{1} p_{2}\right\}$ which implies that $\left[p_{1}^{2}\right]=\left[p_{1}^{4}\right] \in \mathcal{C}(H, F)$. Thus $\left[p_{1}^{2}\right]$ is an idempotent but $p_{1}^{2} \notin H$.

\section{A characterization of half-factoriality}

Let $H$ be a monoid and $\mathcal{A}(H)$ the set of atoms (irreducible elements) of $H$. If an element $a \in H$ has a factorization of the form $a=u_{1} \cdot \ldots \cdot u_{k}$, where $k \in \mathbb{N}$ and $u_{1}, \ldots, u_{k} \in \mathcal{A}(H)$, then $k$ is called a factorization length of $a$. The set $\mathrm{L}_{H}(a)=\mathrm{L}(a)$ of all factorization lengths is called the set of lengths of $a$, and for simplicity we set $\mathrm{L}(a)=\{0\}$ for $a \in H^{\times}$. If $H$ is $v$-noetherian (which holds true of all C-monoids), then every $a \in H$ has a factorization into atoms and all sets of lengths are finite ( [10, Theorem 2.2.9]). For a finite set $L=$ $\left\{m_{0}, \ldots, m_{k}\right\} \subset \mathbb{Z}$, where $k \in \mathbb{N}_{0}$ and $m_{0}<\cdots<m_{k}, \Delta(L)=\left\{m_{i}-m_{i-1} \mid i \in[1, k]\right\}$ is called the set of distances of $L$. Then

$$
\mathcal{L}(H)=\{\mathrm{L}(a) \mid a \in H\} \quad \text { resp. } \Delta(H)=\bigcup_{L \in \mathcal{L}(H)} \Delta(L)
$$

is called the system of sets of lengths resp. the set of distances of $H$. The monoid $H$ is half-factorial if $\Delta(H)=\emptyset$ (equivalently, $|L|=1$ for all $L \in \mathcal{L}(H)$ ).

Half-factoriality has always been a central topic in factorization theory (e.g., [3-5,19,22, 24]). In 1960 Carlitz proved that a ring of integers in an algebraic number field is half-factorial if and only if the class group has at most two elements. This result (which has a simple proof nowadays) carries over to Krull monoids. Indeed, a Krull monoid with class group $G$, that 
has a prime divisor in each class, is half-factorial if and only if $|G| \leq 2$ (the assumption on the distribution of prime divisors is crucial; we refer to [14, Section 5] for background if this assumption fails). We will need this result and the involved machinery in our study of half-factoriality for C-monoids. Thus, we recall that a monoid homomorphism $\theta: H \rightarrow B$ is a transfer homomorphism if the following conditions hold:

(T 1) $B=\theta(H) B^{\times}$and $\theta^{-1}\left(B^{\times}\right)=H^{\times}$.

(T 2) If $u \in H, b, c \in B$ and $\theta(u)=b c$, then there exist $v, w \in H$ such that $u=v w$, $\theta(v) B^{\times}=b B^{\times}$and $\theta(w) B^{\times}=c B^{\times}$.

If $\theta: H \rightarrow B$ is a transfer homomorphism, then $\mathcal{L}(H)=\mathcal{L}(B)$ ([10, Proposition 3.2.3]) whence $H$ is half-factorial if and only if $B$ is half-factorial.

All generalizations of Carlitz's result beyond the setting of Krull monoids have turned out to be surprisingly difficult. We mention two results valid for special classes of C-domains. There is a characterization of half-factoriality for orders in quadratic number fields in number theoretic terms ([10, Theorem 3.7.15]) and for a class of seminormal weakly Krull domains (including seminormal orders in number fields) in algebraic terms involving the $v$-class group and extension properties of prime divisorial ideals (see [11, Theorem 6.2] and [13]).

In this section we establish a characterization of half-factoriality in terms of the class semigroup, that is valid for all seminormal C-monoids and based on Theorem 1.1. Although it is not difficult to show that the set of distances is finite for all C-monoids ([10, Theorem 3.3.4]), a characterization of half-factoriality turns out to be quite involved compared with the simpleness of the result for Krull monoids. But this difference in complexity stems from the fact that the structure of the class semigroup of a $\mathrm{C}$-monoid $H$ can be much more intricate than the structure of the class group $\mathcal{C}(\widehat{H})$ of its complete integral closure. We provide an explicit example of a half-factorial seminormal C-monoid (Example 4.3) and we also refer to the explicit examples of class semigroups given in [20, Section 4].

We introduce our notation which remains valid throughout the rest of this section. Let $H \subset F=F^{\times} \times \mathcal{F}(P)$ be a dense seminormal C-monoid with $\mathrm{E}(\mathcal{C})=\left\{e_{0}=[1], \ldots, e_{n}\right\}$, where $n \in \mathbb{N}$ and $e_{0}+\cdots+e_{n}=e_{n}$. For a subset $T \subset F$, we define

$$
\mathcal{C}_{T}(H, F)=\{[y] \mid y \in T\} \subset \mathcal{C}(H, F) .
$$

We use the abbreviations

$$
\begin{aligned}
\mathcal{C}= & \mathcal{C}(H, F), \mathcal{C}^{*}=\mathcal{C}^{*}(H, F), \mathcal{C}_{H}=\mathcal{C}_{H}(H, F), \mathcal{C}_{i}^{*}=\mathcal{C}_{e_{i}}^{*}, \quad \text { and } \\
& \mathcal{C}_{i}=\mathcal{C}_{e_{i}} \text { for all } i \in[0, n],
\end{aligned}
$$

whence

$$
\begin{gathered}
\mathrm{E}\left(\mathcal{C}^{*}\right)=\mathrm{E}(\mathcal{C}), \quad \mathcal{C}=\biguplus_{i=0}^{n} \mathcal{C}_{i}, \mathcal{C}^{*}=\biguplus_{i=0}^{n} \mathcal{C}_{i}^{*}, \quad \mathcal{C}_{i}=\mathcal{C}_{i}^{*} \text { for all } i \in[1, n], \text { and } \\
\mathcal{C}^{\times}=\mathcal{C}_{0}=\mathcal{C}_{F^{\times}}(H, F) \cup \mathcal{C}_{0}^{*},
\end{gathered}
$$

Proposition 4.1 Let $H \subset F=F^{\times} \times \mathcal{F}(P)$ be a dense seminormal $C$-monoid (with all notations as above) and suppose that every class of $\mathcal{C}^{*}$ contains an element from $P$. For every $i \in[0, n]$, let $P_{i}=\left\{p \in P \mid[p] \in \mathcal{C}_{i}^{*}\right\}, F_{i}=F^{\times} \times \mathcal{F}\left(P_{i}\right), H_{i}=F_{i} \cap H$, and let $\varphi_{i}: \mathcal{C}_{F^{\times}}(H, F) \rightarrow \mathcal{C}_{i}^{*}$ be defined by $\varphi_{i}([\epsilon])=[\epsilon]+e_{i}$ for all $\epsilon \in F^{\times}$. Then for every $k \in[0, n], H_{k} \subset F_{k}$ is a seminormal $C$-monoid and the following statements hold:

1. If $e_{k} \notin \mathcal{C}_{H}$, then $H_{k}=H^{\times}$. If $e_{k} \in \mathcal{C}_{H}$, then

$$
\mathcal{C}\left(H_{k}, F_{k}\right) \cong \begin{cases}\mathcal{C}_{k}, & \text { if } \varphi_{k} \text { is injective, } \\ \mathcal{C}_{F^{\times}}(H, F) \cup \mathcal{C}_{k}, & \text { if } \varphi_{k} \text { is not injective. }\end{cases}
$$


In particular, $\mathcal{C}\left(H_{0}, F_{0}\right) \cong \mathcal{C}_{0}$ and if $\mathcal{C}\left(H_{k}, F_{k}\right)$ is a group, then $H_{k}$ is a Krull monoid. 2. If $e_{k} \in \mathcal{C}_{H}$, then there is a transfer homomorphism $\theta_{k}: H_{k} \rightarrow \mathcal{B}\left(\mathcal{C}_{k}^{*} / \varphi_{k}\left(\mathcal{C}_{F^{\times}}(H, F)\right)\right)$.

Proof Let $k \in[0, n]$. By [10, Proposition 2.9.9], $H_{k} \subset F_{k}$ is a C-monoid and it is a divisorclosed submonoid of $H$. Since $H_{k}$ is a divisor-closed submonoid of the seminormal monoid $H$, it is seminormal (this is easy to check; for details see [11, Lemma 3.2]). By [10, Lemma 2.8.4.5], $\psi_{k}: \mathcal{C}_{F_{k}}(H, F) \rightarrow \mathcal{C}\left(H_{k}, F_{k}\right)$, defined by $\psi_{k}\left([a]_{H}^{F}\right)=[a]_{H_{k}}^{F_{k}}$ for $a \in F_{k}$, is an epimorphism. By definition, we have $\mathcal{C}_{F_{k}}(H, F)=\mathcal{C}_{F^{\times}}(H, F) \cup \mathcal{C}_{k}^{*}=\mathcal{C}_{F^{\times}}(H, F) \cup \mathcal{C}_{k}$.

1. First suppose $e_{k} \notin \mathcal{C}_{H}$. It is clear that $H^{\times} \subset H \cap F_{k}=H_{k}$. Assume to the contrary that there exists an element $a \in H_{k} \backslash H^{\times}$. If $a \in F^{\times}$, then $a \in F^{\times} \cap H=H^{\times}$, a contradiction. If $a \in F_{k} \backslash F^{\times}$, then $[a]_{H}^{F} \in \mathcal{C}_{k}$ and again Theorem 1.1.1 implies that $[a]_{H}^{F}=e_{k} \in \mathcal{C}_{H}$, a contradiction.

Now we suppose that $e_{k} \in \mathcal{C}_{H}$.

1.(a) Suppose that $\varphi_{k}$ is injective. We want to show that $\left.\psi_{k}\right|_{\mathcal{C}_{k}}: \mathcal{C}_{k} \rightarrow \mathcal{C}\left(H_{k}, F_{k}\right)$ is bijective.

First, we show that $\left.\psi_{k}\right|_{\mathcal{C}_{k}}$ is surjective. Let $p \in P_{k}$ such that $[p]_{H}^{F}=e_{k}$ and $\epsilon \in F^{\times}$. Then $p \in H_{k}$. It suffices to prove $[\epsilon]_{H_{k}}^{F_{k}}=[\epsilon p]_{H_{k}}^{F_{k}}$.

Let $a \in F_{k}$. If $\epsilon a \in H_{k}$, then $\epsilon p a \in H_{k}$. Suppose $\epsilon p a \in H_{k}$. Then $[\epsilon p a]_{H}^{F}=[\epsilon a]_{H}^{F}+$ $e_{k}=e_{k}$. If $[\epsilon a]_{H}^{F} \in \mathcal{C}_{k}$, then $[\epsilon a]_{H}^{F}=e_{k}$ and hence $\epsilon a \in H \cap F_{k}=H_{k}$. Suppose $[\epsilon a]_{H}^{F} \in \mathcal{C}_{F^{\times}}(H, F)$. Since $\varphi_{k}$ is injective, we obtain $[\epsilon a]_{H}^{F}=[1]_{H}^{F}$ whence $\epsilon a \in H_{k}$.

In order to show that $\psi_{k} \mid \mathcal{C}_{k}$ is injective, let $p_{1}, p_{2} \in P_{k}$ be given such that $\left[p_{1}\right]_{H}^{F} \neq\left[p_{2}\right]_{H}^{F}$. Since every class of $\mathcal{C}^{*}$ contains an element from $P$, there is a $p \in P_{k}$ such that $[p]_{H}^{F}=$ $-\left[p_{1}\right]_{H}^{F}$. Then $p p_{1} \in H_{k}$ and $p p_{2} \notin H_{k}$ which implies that $\left[p_{1}\right]_{H_{k}}^{F_{k}} \neq\left[p_{2}\right]_{H_{k}}^{F_{k}}$.

1.(b) Suppose that $\varphi_{k}$ is not injective. It suffices to prove $\psi_{k}$ is injective. Let $a_{1}, a_{2} \in F_{k}$ such that $\left[a_{1}\right]_{H}^{F} \neq\left[a_{2}\right]_{H}^{F}$. We have to show that $\left[a_{1}\right]_{H_{k}}^{F_{k}} \neq\left[a_{2}\right]_{H_{k}}^{F_{k}}$ and to do so we distinguish three cases.

If $\left[a_{1}\right]_{H}^{F},\left[a_{2}\right]_{H}^{F} \in \mathcal{C}_{F^{\times}}(H, F)$, then there exists an $\epsilon \in F^{\times}$such that $[\epsilon]_{H}^{F}=-\left[a_{1}\right]_{H}^{F}$. Therefore $\epsilon a_{1} \in H_{k}$ and $\epsilon a_{2} \notin H_{k}$ which imply $\left[a_{1}\right]_{H_{k}}^{F_{k}} \neq\left[a_{2}\right]_{H_{k}}^{F_{k}}$.

If $\left[a_{1}\right]_{H}^{F},\left[a_{2}\right]_{H}^{F} \in \mathcal{C}_{k}$, then there exists an $a \in F_{k}$ such that $[a]_{H}^{F}=-\left[a_{1}\right]_{H}^{F}$. Therefore $a a_{1} \in H_{k}$ and $a a_{2} \notin H_{k}$ which imply $\left[a_{1}\right]_{H_{k}}^{F_{k}} \neq\left[a_{2}\right]_{H_{k}}^{F_{k}}$.

By symmetry, it remains to consider the case where $\left[a_{1}\right]_{H}^{F} \in \mathcal{C}_{k}$ and $\left[a_{2}\right]_{H}^{F} \in \mathcal{C}_{F} \times(H, F)$. Then there exists $\epsilon \in F^{\times}$such that $[\epsilon]_{H}^{F}=-\left[a_{2}\right]_{H}^{F}$. Therefore $\epsilon a_{2} \in H_{k}$. Assume to the contrary that $\left[a_{1}\right]_{H_{k}}^{F_{k}}=\left[a_{2}\right]_{H_{k}}^{F_{k}}$. Then $\epsilon a_{1} \in H_{k}$. Since $\varphi_{k}$ is not injective, there exists $\eta \in F^{\times}$ such that $[\eta]_{H}^{F} \neq[1]_{H}^{F}$ and $[\eta]_{H}^{F}+e_{k}=e_{k}$. Then $\left[\eta \epsilon a_{1}\right]=e_{k}$ whence $\eta \epsilon a_{1} \in H_{k}$ and $\eta \epsilon a_{2} \in H_{k}$. But $\left[\eta \epsilon a_{2}\right]_{H}^{F}=[\eta]_{H}^{F} \neq[1]_{H}^{F}$, a contradiction.

1.(c) Since $\mathcal{C}_{F}(H, F) \cup \mathcal{C}_{0}=\mathcal{C}_{0}$, we have $\mathcal{C}\left(H_{0}, F_{0}\right) \cong \mathcal{C}_{0}$. If $\mathcal{C}\left(H_{k}, F_{k}\right)$ is a group, then $H_{k}$ is a Krull monoid by (2.3) and (2.5).

2. Suppose that $e_{k} \in \mathcal{C}_{H}$. For $g \in \mathcal{C}_{k}^{*}$, we set $\bar{g}=g+\varphi_{k}\left(\mathcal{C}_{F^{\times}}(H, F)\right) \in$ $\mathcal{C}_{k}^{*} / \varphi_{k}\left(\mathcal{C}_{F^{\times}}(H, F)\right)$ and we define

$$
\begin{aligned}
\theta^{*}: F_{k} & \longrightarrow \mathcal{F}\left(\mathcal{C}_{k}^{*} / \varphi_{k}\left(\mathcal{C}_{F^{\times}}(H, F)\right)\right) \\
a=\epsilon \prod_{p \in P_{k}} p^{v_{p}(a)} & \longmapsto \prod_{p \in P_{k}}{\overline{[p]_{H}^{F}}}^{v_{p}(a)} .
\end{aligned}
$$

First, we show that $\theta^{*}\left(H_{k}\right)=\mathcal{B}\left(\mathcal{C}_{k}^{*} / \varphi_{k}\left(\mathcal{C}_{F^{\times}}(H, F)\right)\right)$. Note $H_{k}=H \cap F_{k}=\left\{x \in F_{k} \mid\right.$ $\left.[x]_{H}^{F} \in\left\{e_{0}, e_{k}\right\}\right\}$. If $a \in H_{k}^{\times}$, then $\theta^{*}(a)=1_{\mathcal{F}\left(\mathcal{C}_{k}^{*} / \varphi_{k}\left(\mathcal{C}_{F}(H, F)\right)\right)}$. If $a=\epsilon \prod_{p \in P_{k}} p^{\mathrm{v}_{p}(a)} \in$ $H_{k} \backslash H_{k}^{\times}$, then the sum of the elements of $\theta^{*}(a)$ equals $\sum_{p \in P_{k}} \mathrm{v}_{p}(a) \overline{[p]_{H}^{F}}=\overline{[a]_{H}^{F}}=\overline{e_{k}}$, 
which is the zero element of the group $\mathcal{C}_{k}^{*} / \varphi_{k}\left(\mathcal{C}_{F^{\times}}(H, F)\right)$. Thus we obtain that $\theta^{*}\left(H_{k}\right) \subset$ $\mathcal{B}\left(\mathcal{C}_{k}^{*} / \varphi_{k}\left(\mathcal{C}_{F^{\times}}(H, F)\right)\right)$ and in order to verify equality, we choose an $S=\overline{g_{1}} \cdot \ldots \cdot \overline{g_{\ell}} \in$ $\mathcal{B}\left(\mathcal{C}_{k}^{*} / \varphi_{k}\left(\mathcal{C}_{F^{\times}}(H, F)\right)\right)$, where $g_{1}, \ldots, g_{\ell} \in \mathcal{C}_{k}^{*}$. By assumption, there exist $p_{1}, \ldots, p_{\ell} \in P_{k}$ such that $\left[p_{i}\right]_{H}^{F}=g_{i}$ for all $i \in[1, \ell]$. Since

$$
\overline{\left[p_{1} \cdot \ldots \cdot p_{\ell}\right]_{H}^{F}}=\overline{\left[p_{1}\right]_{H}^{F}}+\cdots+\overline{\left[p_{\ell}\right]_{H}^{F}}=\overline{g_{1}}+\cdots+\overline{g_{\ell}}=0_{\mathcal{C}_{k}^{*} / \varphi_{k}\left(\mathcal{C}_{F} \times(H, F)\right)},
$$

we infer that $\left[p_{1} \cdot \ldots \cdot p_{\ell}\right]_{H}^{F} \in \varphi_{k}\left(\mathcal{C}_{F} \times(H, F)\right)$. Thus there is an $\epsilon \in F^{\times}$such that $\varphi_{k}\left([\epsilon]_{H}^{F}\right)=$ $-\left[p_{1} \cdot \ldots \cdot p_{\ell}\right]_{H}^{F}$. So it follows that $\left[\epsilon p_{1} \cdot \ldots \cdot p_{\ell}\right]_{H}^{F}=e_{k}$ whence $\epsilon p_{1} \cdot \ldots \cdot p_{\ell} \in H \cap F_{k}=H_{k}$ and $\theta^{*}\left(\epsilon p_{1} \cdot \ldots \cdot p_{\ell}\right)=S$.

Secondly, we show $\theta=\left.\theta^{*}\right|_{H_{k}}: H_{k} \rightarrow \mathcal{B}\left(\mathcal{C}_{k}^{*} / \varphi_{k}\left(\mathcal{C}_{F^{*}}(H, F)\right)\right)$ is a transfer homomorphism. Clearly, $\mathcal{B}(\cdot)$ is reduced and (T1) holds. In order to verify (T2), let $a \in H_{k}$ and $b, c \in \mathcal{B}\left(\mathcal{C}_{k}^{*} / \varphi_{k}\left(\mathcal{C}_{F \times}(H, F)\right)\right) \backslash\{1\}$ such that $\theta(a)=b c$. We set $a=\epsilon p_{1} \cdot \ldots \cdot p_{\ell}$, where $\epsilon \in F^{\times}, \ell \in \mathbb{N}_{0}, p_{1}, \ldots, p_{\ell} \in P_{k}$ and, after renumbering if necessary, we assume $b=\overline{\left[p_{1}\right]_{H}^{F}} \cdot \ldots \cdot \overline{\left[p_{s}\right]_{H}^{F}}$ and $c=\overline{\left[p_{s+1}\right]_{H}^{F}} \cdot \ldots \cdot \overline{\left[p_{\ell}\right]_{H}^{F}}$, where $s \in[1, \ell-1]$. Thus there exists an $\epsilon_{1} \in F^{\times}$such that $\varphi_{k}\left(\left[\epsilon_{1}\right]_{H}^{F}\right)=\left[\epsilon_{1}\right]_{H}^{F}+e_{k}=-\left[p_{1} \cdot \ldots \cdot p_{s}\right]_{H}^{F}$ and hence

$$
\begin{aligned}
{\left[\epsilon \epsilon_{1}^{-1}\right]_{H}^{F}+e_{k} } & =[\epsilon]_{H}^{F}+e_{k}-\left[\epsilon_{1}\right]_{H}^{F}+e_{k}=-\left[p_{1} \cdot \ldots \cdot p_{\ell}\right]_{H}^{F}+\left[p_{1} \cdot \ldots \cdot p_{s}\right]_{H}^{F} \\
& =-\left[p_{s+1} \cdot \ldots \cdot p_{\ell}\right]_{H}^{F} .
\end{aligned}
$$

It follows that $u=\epsilon_{1} p_{1} \cdot \ldots \cdot p_{s} \in H_{k}, v=\epsilon \epsilon_{1}^{-1} p_{s+1} \cdot \ldots \cdot p_{\ell} \in H_{k}, a=u v, \theta(u)=b$, and $\theta(v)=c$.

Theorem 4.2 Let $H \subset F=F^{\times} \times \mathcal{F}(P)$ be a dense seminormal $C$-monoid (with all notations as above) and suppose that every class of $\mathcal{C}^{*}$ contains an element from $P$. For every $i \in$ $[0, n]$, let $\varphi_{i}: \mathcal{C}_{F^{\times}}(H, F) \rightarrow \mathcal{C}_{i}^{*}$ be defined by $\varphi_{i}([\epsilon])=[\epsilon]+e_{i}$ for all $\epsilon \in F^{\times}$and set $\mathcal{C}_{i}^{\prime}=\mathcal{C}_{i}^{*} / \varphi_{i}\left(\mathcal{C}_{F^{\times}}(H, F)\right)$. For distinct $i, j \in[0, n]$, let $\phi_{i, j}: \mathcal{C}_{i}^{*} \rightarrow \mathcal{C}_{i}^{*}+\mathcal{C}_{j}^{*}$ be defined by $\phi_{i, j}\left(g_{i}\right)=g_{i}+e_{j}$ for all $g_{i} \in \mathcal{C}_{i}^{*}$.

Then $H$ is half-factorial if and only if the following properties hold:

P1. $\left|\mathcal{C}_{n}^{\prime}\right| \leq 2$.

$P 2$. For every $i \in[0, n]$, we have

$$
\phi_{i, n}^{-1}\left(\varphi_{n}\left(\mathcal{C}_{F^{\times}}(H, F)\right)\right)= \begin{cases}\varphi_{i}\left(\mathcal{C}_{F^{\times}}(H, F)\right), & e_{i} \in \mathcal{C}_{H} \\ \mathcal{C}_{i}, & e_{i} \notin \mathcal{C}_{H}\end{cases}
$$

P3. For distinct $i, j \in[0, n]$, if $e_{i}, e_{j} \in \mathcal{C}_{H}$, then $\operatorname{ker}\left(\phi_{i, j} \circ \varphi_{i}\right)=\operatorname{ker}\left(\varphi_{i}\right)+\operatorname{ker}\left(\varphi_{j}\right)$.

P4. $E\left(\mathcal{C}^{*}\right) \backslash \mathcal{C}_{H}$ is additively closed and for distinct $i_{1}, i_{2}, j \in[0, n]$, if $e_{i_{1}}, e_{i_{2}} \in \mathcal{C}_{H}$ and $e_{j} \in E\left(\mathcal{C}^{*}\right) \backslash \mathcal{C}_{H}$ such that $e_{i_{1}}+e_{i_{2}}+e_{j} \in \mathcal{C}_{H}$, then $e_{i_{1}}+e_{j} \in \mathcal{C}_{H}$ or $e_{i_{2}}+e_{j} \in \mathcal{C}_{H}$.

Proof In order to show that $e_{n} \in \mathcal{C}_{H}$, we choose a $p^{*} \in P$ such that $\left[p^{*}\right]=e_{n}$. Since $H \subset F$ is dense, there exists an $a \in H$ such that $p^{*} \mid a$, say $a=p^{*} b$ with $b \in F$. By Theorem 1.1, $[a]$ is an idempotent and since $[a]=\left[p^{*}\right]+[b]=e_{n}+[b] \in \mathcal{C}_{n}$, it follows that $e_{n}=[a] \in \mathcal{C}_{H}$.

1. We suppose that $H$ is half-factorial and verify properties (P1) - (P4).

(P1). Let $P_{n}=\left\{p \in P \mid[p] \in \mathcal{C}_{n}^{*}\right\}, F_{n}=F^{\times} \times \mathcal{F}\left(P_{n}\right)$, and $H_{n}=H \cap F_{n}$. Since $H_{n}$ is a divisor closed submonoid of $H, H_{n}$ is half-factorial. By Proposition 4.1.3, there is a transfer homomorphism $\theta: H_{n} \rightarrow \mathcal{B}\left(C_{n}^{\prime}\right)$. Thus $\mathcal{B}\left(C_{n}^{\prime}\right)$ is half-factorial which implies that $\left|\mathcal{C}_{n}^{\prime}\right| \leq 2$ ([10, Theorem 3.4.11.5]). 
(P2). Let $i \in[0, n]$ and $e_{i} \in \mathcal{C}_{H}$. Clearly, $e_{i}+e_{n}=e_{n}$ and $\phi_{i, n}\left(\mathcal{C}_{F^{\times}}(H, F)+e_{i}\right)=$ $\mathcal{C}_{F^{\times}}(H, F)+e_{n}$. Assume to the contrary that there exists $g_{i} \in \mathcal{C}_{i}^{*}$ with $g_{i} \notin$ $\varphi_{i}\left(\mathcal{C}_{F^{\times}}(H, F)\right)$ such that $\phi_{i, n}\left(g_{i}\right) \in \mathcal{C}_{F^{\times}}(H, F)+e_{n}$, i.e. there exists $\epsilon \in F^{\times}$such that $g_{i}+e_{n}=[\epsilon]+e_{n}$. Let $p_{1}, p_{2}, q \in P$ such that $\left[p_{1}\right]=g_{i},\left[p_{2}\right]=-g_{i}$, and $[q]=e_{n}$. Then $\left[\epsilon^{-1} p_{1} q\right]=\left[\epsilon p_{2} q\right]=[q]=e_{n}$ and $\left[p_{1} p_{2}\right]=e_{i}$. We claim that $\epsilon^{-1} p_{1} q, \epsilon p_{2} q, p_{1} p_{2}, q \in \mathcal{A}(H)$. Clearly, the elements lie in $H$ and, for example, assume to the contrary that $p_{1} p_{2}$ is not an atom. Then there is an $\eta \in F^{\times}$such that $\eta p_{1}, \eta^{-1} p_{2} \in H$. Then $\left[\eta p_{1}\right]=[\eta]+\left[p_{1}\right]=e_{i}$ whence $g_{i}=\left[p_{1}\right]=\left[\eta^{-1}\right]+e_{i} \in \varphi_{i}\left(\mathcal{C}_{F^{\times}}(H, F)\right)$, a contradiction. Thus we obtain that $\left(p_{1} p_{2}\right)(q)^{2}=\left(\epsilon^{-1} p_{1} q\right)\left(\epsilon p_{2} q\right)$, a contradiction to the half-factoriality of $H$.

Suppose $e_{i} \in \mathrm{E}\left(\mathcal{C}^{*}\right) \backslash \mathcal{C}_{H}$. Assume to the contrary that there exists $g_{i} \in \mathcal{C}_{i}$ such that $\phi_{i, n}\left(g_{i}\right)=g_{i}+e_{n} \notin \mathcal{C}_{F}(H, F)+e_{n}$. Let $p_{1}, p_{2}, p_{3} \in P$ such that $\left[p_{1}\right]=g_{i}$, $\left[p_{2}\right]=e_{n}$, and $\left[p_{3}\right]=-g_{i}+e_{n}$, and let ord $\left(g_{i}\right)=\operatorname{ord}_{\mathcal{C}_{i}}\left(g_{i}\right)$ denote the order of $g_{i}$ in the group $\mathcal{C}_{i}$. Then $\left[p_{1}^{\operatorname{ord}\left(g_{i}\right)} p_{2}\right]=\left[p_{2}\right]=\left[p_{1} p_{3}\right]=e_{n} \in \mathcal{C}_{H}$ which implies that $p_{1}^{\operatorname{ord}\left(g_{i}\right)} p_{2}, p_{2}, p_{1} p_{3} \in \mathcal{A}(H)$. Therefore $\left(p_{1} p_{3}\right)^{\operatorname{ord}\left(g_{i}\right)} p_{2}=\left(p_{1}^{\operatorname{ord}\left(g_{i}\right)} p_{2}\right)\left(p_{3}^{\operatorname{ord}\left(g_{i}\right)}\right)$. Since $p_{3}^{\operatorname{ord}\left(g_{i}\right)} \in H$ and for any $\epsilon \in F^{\times}, \epsilon p_{3} \notin H$, we obtain $\operatorname{ord}\left(g_{i}\right) \notin \mathrm{L}_{H}\left(p_{3}^{\operatorname{ord}\left(g_{i}\right)}\right)$, a contradiction to the half-factoriality of $H$.

(P3). Let $i, j \in[0, n]$ be distinct and $e_{i}, e_{j} \in \mathcal{C}_{H}$. Since $\phi_{i, j} \circ \varphi_{i}=\phi_{j, i} \circ \varphi_{j}$, we have $\operatorname{ker}\left(\phi_{i, j} \circ \varphi_{i}\right) \supset \operatorname{ker}\left(\varphi_{i}\right)$ and $\operatorname{ker}\left(\phi_{i, j} \circ \varphi_{i}\right) \supset \operatorname{ker}\left(\varphi_{j}\right)$ which $\operatorname{imply} \operatorname{ker}\left(\phi_{i, j} \circ \varphi_{i}\right) \supset$ $\operatorname{ker}\left(\varphi_{i}\right)+\operatorname{ker}\left(\varphi_{j}\right)$. Let $\epsilon \in F^{\times}$such that $\phi_{i, j}\left(\varphi_{i}([\epsilon])\right)=e_{i}+e_{j}$. If $[\epsilon]+e_{i}=e_{i}$ or $[\epsilon]+e_{j}=e_{j}$, then $[\epsilon]=[\epsilon]+[1] \in \operatorname{ker}\left(\varphi_{i}\right)+\operatorname{ker}\left(\varphi_{j}\right)$. Suppose $[\epsilon]+e_{i} \neq e_{i}$ and $[\epsilon]+$ $e_{j} \neq e_{j}$. Let $p_{1}, p_{2}, p_{3} \in P$ such that $\left[p_{1}\right]=[\epsilon]+e_{i},\left[p_{2}\right]=e_{j}$, and $\left[p_{3}\right]=e_{i}+e_{j}$. Since $p_{2}, p_{3}, \epsilon p_{3}, \epsilon^{-1} p_{1} \in \mathcal{A}(H)$, the equation $\left(p_{1} p_{2}\right) p_{3}=\left(\epsilon^{-1} p_{1}\right)\left(\epsilon p_{3}\right) p_{2}$ implies that $p_{1} p_{2} \notin \mathcal{A}(H)$. Then there exists $\delta \in F^{\times}$such that $\delta p_{1} \in H$ and $\delta^{-1} p_{2} \in H$ whence $\left[\delta p_{1}\right]=[\delta]+[\epsilon]+e_{i}=e_{i}$ and $\left[\delta^{-1} p_{2}\right]=-[\delta]+e_{j}=e_{j}$. It follows that $[\delta]+[\epsilon] \in \operatorname{ker}\left(\varphi_{i}\right),-[\delta] \in \operatorname{ker}\left(\varphi_{j}\right)$, and $[\epsilon]=[\delta]+[\epsilon]-[\delta] \in \operatorname{ker}\left(\varphi_{i}\right)+\operatorname{ker}\left(\varphi_{j}\right)$.

(P4). Assume to the contrary that there exist $f_{1}, f_{2} \in \mathrm{E}\left(\mathcal{C}^{*}\right) \backslash \mathcal{C}_{H}$ such that $f_{1}+f_{2} \in \mathcal{C}_{H}$. Let $q_{1}, q_{2} \in P$ such that $\left[q_{1}\right]=f_{1}$ and $\left[q_{2}\right]=f_{2}$. Then $\left[q_{1} q_{2}\right]=\left[q_{1}^{2} q_{2}\right]=\left[q_{1} q_{2}^{2}\right]=$ $f_{1}+f_{2} \in \mathcal{C}_{H}, q_{1} q_{2}, q_{1}^{2} q_{2}, q_{1} q_{2}^{2} \in \mathcal{A}(H)$, and $\left(q_{1} q_{2}\right)^{3}=\left(q_{1}^{2} q_{2}\right)\left(q_{1} q_{2}^{2}\right)$, a contradiction to the half-factoriality of $H$.

Let $i_{1}, i_{2}, j \in[0, n]$ such that $e_{j} \in \mathrm{E}\left(\mathcal{C}^{*}\right) \backslash \mathcal{C}_{H}$ and $e_{i_{1}}, e_{i_{2}}, e_{i_{1}}+e_{i_{2}}+e_{j} \in \mathcal{C}_{H}$. Assume to the contrary that $e_{i_{1}}+e_{j}, e_{i_{2}}+e_{j} \in \mathrm{E}\left(\mathcal{C}^{*}\right) \backslash \mathcal{C}_{H}$. Let $p, p_{1}, p_{2}, p_{3} \in P$ such that $[p]=e_{i_{1}}+e_{i_{2}},\left[p_{1}\right]=e_{i_{1}},\left[p_{2}\right]=e_{i_{2}},\left[p_{3}\right]=e_{j}$. Then $p, p_{1}, p_{2}, p p_{3}, p_{1} p_{2} p_{3} \in$ $\mathcal{A}(H)$ and $(p)\left(p_{1} p_{2} p_{3}\right)=\left(p_{1}\right)\left(p_{2}\right)\left(p p_{3}\right)$, a contradiction to the half-factoriality of $H$.

2. We suppose that (P1) - (P4) hold and prove that $H$ is half-factorial. We start with the following three assertions.

A1. If $i \in[0, n]$ and $e_{i} \in \mathcal{C}_{H}$, then $\left|\mathcal{C}_{i}^{\prime}\right| \leq 2$.

A2. If $i, j \in[0, n]$ and $e_{i}, e_{j} \in \mathcal{C}_{H}$, then $\phi_{i, j}^{-1}\left(\mathcal{C}_{F^{\times}}(H, F)+e_{i}+e_{j}\right)=\mathcal{C}_{F^{\times}}(H, F)+e_{i}$.

A3. If $i, j \in[0, n], e_{i} \notin \mathcal{C}_{H}$, and $e_{j} \in \mathcal{C}_{H}$ such that $e_{i}+e_{j} \in \mathcal{C}_{H}$, then $\phi_{i, j}^{-1}\left(\mathcal{C}_{F^{\times}}(H, F)+\right.$ $\left.e_{i}+e_{j}\right)=\mathcal{C}_{i}$.

Proof of A1. Let $i \in[0, n]$ and $e_{i} \in \mathcal{C}_{H}$. By (P2), there is a monomorphism $\phi_{i, n}^{\prime}: \mathcal{C}_{i}^{\prime} \rightarrow \mathcal{C}_{n}^{\prime}$ defined by $\phi_{i, n}^{\prime}\left(h+\varphi_{i}\left(\mathcal{C}_{F^{\times}}(H, F)\right)\right)=h+e_{n}+\varphi_{n}\left(\mathcal{C}_{F^{\times}}(H, F)\right)$ for all $h \in \mathcal{C}_{i}^{*}$. It follows by (P1) that $\left|\mathcal{C}_{i}^{\prime}\right| \leq 2$.

Proof of A2. Let $i, j \in[0, n]$ and $e_{i}, e_{j} \in \mathcal{C}_{H}$, say $e_{i}+e_{j}=e_{k}$ with $k \in[0, n]$, and note that $\phi_{i, n}=\phi_{k, n} \circ \phi_{i, k}$ and $\phi_{i, j}=\phi_{i, k}$. Then (P2) implies that $\mathcal{C}_{F \times}(H, F)+e_{i}=$ $\phi_{i, n}^{-1}\left(\mathcal{C}_{F^{\times}}(H, F)+e_{n}\right)=\phi_{i, k}^{-1}\left(\phi_{k, n}^{-1}\left(\mathcal{C}_{F^{\times}}(H, F)+e_{n}\right)\right)=\phi_{i, j}^{-1}\left(\mathcal{C}_{F^{\times}}(H, F)+e_{k}\right)$. 
Proof of A3. Let $i, j \in[0, n], e_{i} \in \mathrm{E}\left(\mathcal{C}^{*}\right) \backslash \mathcal{C}_{H}$, and $e_{j} \in \mathcal{C}_{H}$ such that $e_{i}+e_{j} \in \mathcal{C}_{H}$, say $e_{i}+e_{j}=e_{k}$ with $k \in[0, n]$, and again we note that $\phi_{i, n}=\phi_{k, n} \circ \phi_{i, k}$ and $\phi_{i, j}=\phi_{i, k}$. Then (P2) implies that $\mathcal{C}_{i}=\phi_{i, n}^{-1}\left(\mathcal{C}_{F^{\times}}(H, F)+e_{n}\right)=\phi_{i, k}^{-1}\left(\phi_{k, n}^{-1}\left(\mathcal{C}_{F^{\times}}(H, F)+e_{n}\right)\right)=$ $\phi_{i, j}^{-1}\left(\mathcal{C}_{F \times}(H, F)+e_{k}\right)$.

Now we set $I=\left\{i \in[0, n] \mid e_{i} \in \mathcal{C}_{H}\right\}$ and we define

$$
G^{1}=\bigcup_{i \in I} \varphi_{i}\left(\mathcal{C}_{F^{\times}}(H, F)\right), G^{2}=\bigcup_{i \in I}\left(\mathcal{C}_{i}^{*} \backslash \varphi_{i}\left(\mathcal{C}_{F^{\times}}(H, F)\right)\right), \text { and } G^{3}=\mathcal{C}^{*} \backslash \bigcup_{i \in I} \mathcal{C}_{i}^{*}
$$

For every $a=\epsilon p_{1} \cdot \ldots \cdot p_{\ell} \in F$, where $\epsilon \in F^{\times}, \ell \in \mathbb{N}_{0}$, and $p_{1}, \ldots, p_{\ell} \in P$, we define

$$
\begin{aligned}
\mathrm{I}_{1}(a)= & \left|\left\{j \in[1, \ell] \mid\left[p_{j}\right] \in G^{1}\right\}\right|, \mathrm{I}_{2}(a)=\left|\left\{k \in[1, \ell] \mid\left[p_{k}\right] \in G^{2}\right\}\right|, \text { and } \\
& \mathrm{I}(a)=\mathrm{I}_{1}(a)+\frac{1}{2} \mathrm{I}_{2}(a) .
\end{aligned}
$$

In order to prove that $H$ is half-factorial, it is sufficient to prove the following assertion.

A4. If $a$ is an atom of $H$, then $\mathrm{I}(a)=1$.

Proof of A4. Let $a=\epsilon p_{1} \cdot \ldots \cdot p_{\ell} \in F \backslash F^{\times}$be an atom of $H$. Assume to the contrary that $\mathrm{I}(a)=0$. Then, for every $i \in[1, \ell]$, we have $\left[p_{i}\right] \in G^{3}$, say $\left[p_{i}\right] \in \mathcal{C}_{f_{i}}$ with $f_{i} \in \mathrm{E}\left(\mathcal{C}^{*}\right) \backslash \mathcal{C}_{H}$. Clearly, we have $[a]=[\epsilon]+\left[p_{1}\right]+\cdots+\left[p_{\ell}\right] \in \mathcal{C}_{[a]}$ and $[a] \in \mathcal{C}_{F} \times(H, F)+\mathcal{C}_{f_{1}}+\cdots+\mathcal{C}_{f_{\ell}} \subset$ $\mathcal{C}_{f_{1}+\cdots+f_{\ell}}$ whence $f_{1}+\cdots+f_{\ell}=[a]$, a contradiction to (P4).

Assume to the contrary that $\mathrm{I}(a) \geq 2$ and distinguish three cases.

CASE 1: $I_{1}(a) \geq 2$.

After renumbering if necessary, we may assume that $\left[p_{1}\right],\left[p_{2}\right] \in G^{1}$. Then there exists $\epsilon_{1}, \epsilon_{2} \in F^{\times}$and $e_{i}, e_{j} \in \mathcal{C}_{H}$ such that $\left[p_{1}\right]=\left[\epsilon_{1}\right]+e_{i},\left[p_{2}\right]=\left[\epsilon_{2}\right]+e_{j}$. We set $g=$ $\left[\epsilon p_{3} \cdot \ldots \cdot p_{\ell}\right] \in \mathcal{C}_{k}$ and $[a]=e_{r} \in \mathcal{C}_{H}$ for some $k, r \in[0, n]$. Then

$$
e_{r}=\left[\epsilon_{1}\right]+e_{i}+\left[\epsilon_{2}\right]+e_{j}+g \in \mathcal{C}_{F \times}(H, F)+\mathcal{C}_{i}+\mathcal{C}_{j}+\mathcal{C}_{k} \subset \mathcal{C}_{e_{i}+e_{j}+e_{k}}
$$

whence $e_{i}+e_{j}+e_{k}=e_{r} \in \mathcal{C}_{H}$. By (P4), we obtain that $e_{i}+e_{k} \in \mathcal{C}_{H}$ or $e_{j}+e_{k} \in \mathcal{C}_{H}$, say $e_{j}+e_{k}=e_{t} \in \mathcal{C}_{H}$ for some $t \in[0, n]$.

Since $\left[\epsilon_{1}\right]+\left[p_{2}\right]+g \in \mathcal{C}_{t}$ and

$$
\begin{aligned}
\phi_{t, i}\left(\left[\epsilon_{1}\right]+\left[p_{2}\right]+g\right) & =\left[\epsilon_{1}\right]+\left[p_{2}\right]+g+e_{i}=\left[p_{1}\right]+\left[p_{2}\right]+g=\left[\epsilon p_{1} \cdot \ldots \cdot p_{\ell}\right]=e_{r} \\
& =e_{i}+e_{t} \in \mathcal{C}_{F \times}(H, F)+e_{i}+e_{t},
\end{aligned}
$$

we obtain $\left[\epsilon_{1}\right]+\left[p_{2}\right]+g \in \mathcal{C}_{F^{\times}}(H, F)+e_{t}$ by $\mathbf{A 2}$. It follows that $\left[\epsilon p_{2} \cdot \ldots \cdot p_{\ell}\right]=\left[p_{2}\right]+g \in$ $\mathcal{C}_{F^{\times}}(H, F)+e_{t}$ whence there exists $\epsilon_{3} \in F^{\times}$such that $\left[\epsilon p_{2} \cdot \ldots \cdot p_{\ell}\right]=\left[\epsilon_{3}\right]+e_{t}$. Since

$$
e_{i}+e_{t}=e_{r}=[a]=\left[p_{1}\right]+\left[\epsilon p_{2} \cdot \ldots \cdot p_{\ell}\right]=\left[\epsilon_{1}\right]+e_{i}+\left[\epsilon_{3}\right]+e_{t},
$$

we infer that $\left[\epsilon_{1}\right]+\left[\epsilon_{3}\right] \in \operatorname{ker}\left(\phi_{i, t} \circ \varphi_{i}\right)$. By $(\mathrm{P} 3)$, there exists $\delta \in F^{\times}$such that $[\delta] \in \operatorname{ker}\left(\varphi_{i}\right)$ and $\left[\epsilon_{1} \epsilon_{3} \delta^{-1}\right] \in \operatorname{ker}\left(\varphi_{t}\right)$. Thus we obtain that

$$
\begin{aligned}
& {\left[\delta \epsilon_{1}^{-1} p_{1}\right]=[\delta]-\left[\epsilon_{1}\right]+\left[p_{1}\right]=[\delta]+e_{i}=e_{i}} \\
& {\left[\epsilon_{1} \delta^{-1} \epsilon p_{2} \cdot \ldots \cdot p_{\ell}\right]=\left[\epsilon_{1} \epsilon_{3} \delta^{-1} \epsilon_{3}^{-1} \epsilon p_{2} \cdot \ldots \cdot p_{\ell}\right]=\left[\epsilon_{1} \epsilon_{3} \delta^{-1}\right]-\left[\epsilon_{3}\right]} \\
& \quad+\left[\epsilon p_{2} \cdot \ldots \cdot p_{\ell}\right]=\left[\epsilon_{1} \epsilon_{2} \delta^{-1}\right]+e_{t}=e_{t} .
\end{aligned}
$$

Thus $\delta \epsilon_{1}^{-1} p_{1} \in H \backslash H^{\times}, \epsilon_{1} \delta^{-1} \epsilon p_{2} \cdot \ldots \cdot p_{\ell} \in H \backslash H^{\times}$, and $a=\left(\delta \epsilon_{1}^{-1} p_{1}\right)\left(\epsilon_{1} \delta^{-1} \epsilon p_{2} \cdot \ldots \cdot p_{\ell}\right)$, a contradiction to $a \in \mathcal{A}(H)$. 
CASE 2: $\mathrm{I}_{1}(a)=1$ and $\mathrm{I}_{2}(a) \geq 2$.

After renumbering if necessary, we may assume that $\left[p_{1}\right] \in G^{1}$, and $\left[p_{2}\right],\left[p_{3}\right] \in G^{2}$. There are $i, j, k \in[0, n]$ such that $\left[p_{2}\right] \in \mathcal{C}_{i}^{*} \backslash \varphi_{i}\left(\mathcal{C}_{F^{\times}}(H, F),\left[p_{3}\right] \in \mathcal{C}_{j}^{*} \backslash \varphi_{j}\left(\mathcal{C}_{F^{\times}}(H, F)\right.\right.$, and $e_{k}=e_{i}+e_{j}$. By $\mathbf{A} 2$ we infer that

$$
\phi_{i, k}\left(\left[p_{2}\right]\right)=\left[p_{2}\right]+e_{k} \notin \mathcal{C}_{F^{\times}}(H, F)+e_{i}+e_{k}=\varphi_{k}\left(\mathcal{C}_{F^{\times}}(H, F)\right)
$$

and

$$
\phi_{i, k}\left(\left[p_{3}\right]\right)=\left[p_{3}\right]+e_{k} \notin \mathcal{C}_{F^{\times}}(H, F)+e_{i}+e_{k}=\varphi_{k}\left(\mathcal{C}_{F^{\times}}(H, F)\right) .
$$

Since $\left[p_{2}\right]+e_{k}$ and $\left[p_{3}\right]+e_{k}$ are in $\mathcal{C}_{k}^{*} \backslash \varphi_{k}\left(\mathcal{C}_{F^{\times}}(H, F)\right)$ and since, by $\mathbf{A 1},\left|\mathcal{C}_{k}^{\prime}\right| \leq 2$, it follows that $\left[p_{2}\right]+\left[p_{3}\right]=\left(\left[p_{2}\right]+e_{k}\right)+\left(\left[p_{3}\right]+e_{k}\right) \in \varphi_{k}\left(\mathcal{C}_{F \times}(H, F)\right) \subset G^{1}$. We choose a $q \in P$ such that $[q]=\left[p_{2}\right]+\left[p_{3}\right]$. Then $b=\epsilon p_{1} q p_{4} \cdot \ldots \cdot p_{\ell} \in \mathcal{A}(H)$ with $\mathrm{I}(b)=\mathrm{I}(a) \geq 2$. Now we are back to CASE 1.

CASE 3: $\mathrm{I}_{1}(a)=0$ and $\mathrm{I}_{2}(a) \geq 4$.

After renumbering if necessary, we may assume that $\left[p_{1}\right],\left[p_{2}\right],\left[p_{3}\right],\left[p_{4}\right] \in G^{2}$. Arguing as in CASE 2 we infer that $\left[p_{1}\right]+\left[p_{2}\right],\left[p_{3}\right]+\left[p_{4}\right] \in G^{1}$. Let $q_{1}, q_{2} \in P$ such that $\left[q_{1}\right]=\left[p_{1}\right]+\left[p_{2}\right]$ and $\left[q_{2}\right]=\left[p_{3}\right]+\left[p_{4}\right]$. Then $b=\epsilon q_{1} q_{2} p_{4} \cdot \ldots \cdot p_{\ell} \in \mathcal{A}(H)$ with $\mathrm{I}(b)=\mathrm{I}(a) \geq 2$ whence we are back to CASE 1 .

The following example shows that both the number and the size of the constituent groups of the reduced class semigroup of a half-factorial seminormal C-monoid can be arbitrarily large.

Example 4.3 Let $C$ be a Clifford semigroup with

$$
\mathrm{E}(C)=\left\{e_{0}, e_{1}, \ldots, e_{n}\right\}, C=\biguplus_{i=0}^{n} C_{i}, \quad \text { and } \quad C_{e_{i}}=C_{i}=G_{i} \oplus \mathbb{Z} / 2 \mathbb{Z},
$$

where $n \in \mathbb{N}, i, j \in[0, n], C_{i}+e_{j}=C_{j}$ whenever $i<j$, and $G_{0} \supsetneq \cdots \supsetneq G_{n}=\{1\}$ are abelian groups. Let

$$
F=F^{\times} \times \mathcal{F}(\mathcal{C}) \text { and } B=\{\epsilon S \in F \mid \iota(\epsilon)+\sigma(S) \in \mathrm{E}(C)\},
$$

where $F^{\times}=G_{0}, \iota: F^{\times} \rightarrow C_{0}$ is a monomorphism, and $\sigma: \mathcal{F}(\mathcal{C}) \rightarrow \mathcal{C}$ is the sum function, which is defined as $\sigma\left(g_{1} \cdot \ldots \cdot g_{\ell}\right)=g_{1}+\cdots+g_{\ell}$. Then $B \subset F$ is a half-factorial dense seminormal C-monoid such that every class of $\mathcal{C}(B, F)$ contains an element from $C$,

$$
\mathcal{C}^{*}(B, F)=\mathcal{C}(B, F) \cong C, \mathcal{C}_{F^{\times}}(B, F) \cong F^{\times}, \quad \text { and } \mathcal{C}_{B}(B, F)=\mathrm{E}\left(\mathcal{C}^{*}(B, F)\right) .
$$

Proof We start with the following four assertions. Let $\epsilon, \epsilon_{1}, \epsilon_{2} \in F^{\times}, S, S_{1}, S_{2} \in \mathcal{F}(\mathcal{C})$, and consider the epimorphism

$$
\lambda: F^{\times} \times \mathcal{F}(C) \rightarrow C \text { defined by } \lambda(\epsilon S)=\iota(\epsilon)+\sigma(S) \text { for all } \epsilon S \in F^{\times} \times \mathcal{F}(C) .
$$

A1. $\epsilon_{1} S_{1} \sim \epsilon_{2} S_{2}$ if and only if $\lambda\left(\epsilon_{1} S_{1}\right)=\lambda\left(\epsilon_{2} S_{2}\right)$. In particular, $[\epsilon S]=[\lambda(\epsilon S)]$.

A2. The map $\psi: \mathcal{C}^{*}(B, F) \rightarrow \mathcal{C}$, defined by $[\epsilon S] \mapsto \lambda(\epsilon S)$, is a semigroup isomorphism.

A3. $\mathcal{C}_{F^{*}}(B, F) \cong F^{\times}$and $\mathcal{C}^{*}(B, F)=\mathcal{C}(B, F)$.

A4. $\mathcal{C}_{B}(B, F)=\mathrm{E}\left(\mathcal{C}^{*}(B, F)\right)$.

Suppose these four statements hold true. Since $B^{\times}=\{1\}=F^{\times} \cap B, \mathbf{A 2}$ implies that $B \subset F$ is a C-monoid. Clearly, $B \subset F$ is dense and by Theorem $1.1 B$ is seminormal. Since every class of $\mathcal{C}^{*}(B, F)$ contains a prime from $C$, all assumptions of Theorem 4.2 are satisfied and we obtain that $H$ is half-factorial. 
Proof of A1. By definition of $B, \lambda\left(\epsilon_{1} S_{1}\right)=\lambda\left(\epsilon_{2} S_{2}\right)$ implies that $\epsilon_{1} S_{1} \sim \epsilon_{2} S_{2}$. Conversely, suppose $\epsilon_{1} S_{1} \sim \epsilon_{2} S_{2}$ and $\lambda\left(\epsilon_{1} S_{1}\right) \neq \lambda\left(\epsilon_{2} S_{2}\right)$. If there exists an $i \in[0, n]$ such that $\lambda\left(\epsilon_{1} S_{1}\right), \lambda\left(\epsilon_{2} S_{2}\right) \in C_{i}$, then $a=-\lambda\left(\epsilon_{1} S_{1}\right) \in \mathcal{C} \subset F$ and hence $\epsilon_{1} S_{1} a \in B, \epsilon_{2} S_{2} a \notin B$, a contradiction. After renumbering if necessary we may assume that $\lambda\left(\epsilon_{1} S_{1}\right) \in C_{i}, \lambda\left(\epsilon_{2} S_{2}\right) \in$ $C_{j}$ for some $i, j \in[0, n]$ with $i<j$. Let $a=-\lambda\left(\epsilon_{1} S_{1}\right) \in \mathcal{C} \subset F$. Since $G_{i} \supsetneq G_{j}$ and $C_{i}+e_{j}=C_{j}$, there exists $g_{i} \in C_{i} \backslash\left\{e_{i}\right\}$ such that $g_{i}+e_{j}=e_{j}$. Then $\epsilon_{1} S_{1} a \in B$ which implies that $\epsilon_{2} S_{2} a \in B$. Thus $\epsilon_{2} S_{2} a g_{i} \in B$ which implies that $\epsilon_{1} S_{1} a g_{i} \in B$. But $\lambda\left(\epsilon_{1} S_{1} a g_{i}\right)=g_{i} \neq e_{i}$, a contradiction.

To verify the in particular statement, note that $\lambda(\epsilon S)=\iota(\epsilon)+\sigma(S) \in \mathcal{C} \subset F$ and for an element $g \in \mathcal{C}$ we have $\lambda(g)=g$. Thus the claim follows immediately from the main statement.

Proof of A2. By A1, $\psi$ is a well-defined monomorphism and obviously $\psi$ is surjective.

Proof of A3. Since $\iota: F^{\times} \rightarrow C_{0}$ is a monomorphism, we have, using (2.2), that $F^{\times}=$ $F^{\times} / B^{\times} \cong \mathcal{C}_{F^{\times}}(B, F) \subset C_{0}$ which implies that $\mathcal{C}(B, F)=\mathcal{C}^{*}(B, F)$.

Proof of A4. Theorem 1.1 implies that $\mathcal{C}_{B}(B, F) \subset \mathrm{E}\left(\mathcal{C}^{*}(B, F)\right)$. Conversely, let $\epsilon S \in F$ such that $[\epsilon S] \in \mathrm{E}\left(\mathcal{C}^{*}(B, F)\right)$. Then $\mathbf{A} 2$ implies that $\iota(\epsilon)+\sigma(S) \in \mathrm{E}(\mathcal{C})$. Thus, by the definition of $B$, it follows that $\epsilon S \in B$ whence $[\epsilon S] \in \mathcal{C}_{B}(B, F)$.

Acknowledgements Open access funding provided by Austrian Science Fund (FWF). We thank the referee for their careful reading and all their comments.

Open Access This article is distributed under the terms of the Creative Commons Attribution 4.0 International License (http://creativecommons.org/licenses/by/4.0/), which permits unrestricted use, distribution, and reproduction in any medium, provided you give appropriate credit to the original author(s) and the source, provide a link to the Creative Commons license, and indicate if changes were made.

\section{References}

1. Baginski, P., Chapman, S.T.: Congruence, arithmetic, monoids: a survey, combinatorial and additive number theory: CANT. 2012, Proceedings in Mathematics and Statistics, vol. 101, pp. 15-38. Springer, Berlin (2011)

2. Barucci, V.: Seminormal Mori domains, Commutative Ring Theory. Lect. Notes Pure Appl. Math., vol. 153, pp. 1-12. Marcel Dekker, New York City (1994)

3. Chapman, S.T., Coykendall, J.: Half-Factorial Domains, A Survey, Non-Noetherian Commutative Ring Theory, Mathematics and Its Applications, vol. 520, pp. 97-115. Kluwer Academic, Alphen aan den Rijn (2000)

4. Coykendall, J.: Extensions of half-factorial domains : a survey, Arithmetical Properties of Commutative Math Rings and Monoids, Lect. Notes Pure Appl, vol. 241, pp. 46-70. Chapman \& Hall/CRC, Oxford (2005)

5. Coykendall, J., Malcolmson, P., Okoh, F.: Inert primes and factorization in extensions of quadratic orders. Houston J. Math. 43, 61-77 (2017)

6. Cziszter, K., Domokos, M., Geroldinger, A.: The Interplay of Invariant Theory with Multiplicative Ideal Theory and with Arithmetic Combinatorics, Multiplicative Ideal Theory and Factorization Theory, pp. 43-95. Springer, Berlin (2016)

7. Dobbs, D.E., Fontana, M.: Seminormal rings generated by algebraic integers. Mathematika 34, 141-154 (1987)

8. Foroutan, A., Hassler, W.: Factorization of powers in C-monoids. J. Algebra 304, 755-781 (2006)

9. Geroldinger, A., Halter-Koch, F.: Congruence monoids. Acta Arith. 112, 263-296 (2004)

10. Geroldinger, A., Halter-Koch, F.: Non-Unique Factorizations. Algebraic, Combinatorial and Analytic Theory, Pure and Applied Mathematics, vol. 278. Chapman \& Hall/CRC, Oxford (2006)

11. Geroldinger, A., Kainrath, F., Reinhart, A.: Arithmetic of seminormal weakly Krull monoids and domains. J. Algebra 444, 201-245 (2015)

12. Geroldinger, A., Ramacher, S., Reinhart, A.: On $v$-Marot Mori rings and C-rings. J. Korean Math. Soc. 52, 1-21 (2015) 
13. Geroldinger, A., Zhong, Q.: The set of distances in seminormal weakly Krull monoids. J. Pure Appl. Algebra 220, 3713-3732 (2016)

14. Gilmer, R.: Some questions for further research, Multiplicative Ideal Theory in Commutative Algebra, pp. 405-415. Springer, New York (2006)

15. Grillet, P.A.: Commutative Semigroups. Kluwer Academic, Alphen aan den Rijn (2001)

16. Halter-Koch, F.: Clifford semigroups of ideals in monoids and domains. Forum Math. 21, 1001-1020 (2009)

17. Halter-Koch, F., Hassler, W., Kainrath, F.: Remarks on the Multiplicative Structure of Certain OneDimensional Integral Domains, Rings, Modules, Algebras, and Abelian Groups, Lect. Notes Pure Appl. Math., vol. 236, pp. 321-331. Marcel Dekker, New York City (2004)

18. Kainrath, F.: Arithmetic of Mori Domains and Monoids: The Global Case, Multiplicative Ideal Theory and Factorization Theory, Springer Proc. Math. Stat., vol. 170, pp. 183-218. Springer, Berlin (2016)

19. Malcolmson, P., Okoh, F.: Half-factorial subrings of factorial domains. J. Pure Appl. Algebra 220, 877891 (2016)

20. Oh, J.: On the algebraic and arithmetic structure of the monoid of product-one sequences. J. Commut. Algebra (to appear). https://projecteuclid.org/euclid.jca/1523433705

21. Oh, J.: On the algebraic and arithmetic structure of the monoid of product-one sequences II, Periodica Math. Hungarica (to appear). arxiv:1802.02851

22. Philipp, A.: A precise result on the arithmetic of non-principal orders in algebraic number fields. J. Algebra Appl. 11, 1250087 (2012). 42pp

23. Reinhart, A.: On integral domains that are C-monoids. Houston J. Math. 39, 1095-1116 (2013)

24. Schmid, W.A.: Half-factorial sets in finite abelian groups: a survey. Grazer Math. Ber. 348, 41-64 (2005)

25. Schmid, W.A.: Some Recent Results and Open Problems on Sets of Lengths of Krull Monoids with Finite Class Group, Multiplicative Ideal Theory and Factorization Theory, Springer Proc. Math. Stat., vol. 170, pp. 323-352. Springer, Berlin (2016)

Publisher's Note Springer Nature remains neutral with regard to jurisdictional claims in published maps and institutional affiliations. 